\title{
CHOLINESTERASE IN NORMAL AND ABNORMAL HUMAN SKELETAL MUSCLE
}

\author{
BY \\ EVELYN B. BECKETT and G. H. BOURNE \\ From the Department of Neurology, the London Hospital, and the Department of Histology, \\ London Hospital Medical College
}

The original theory of neuromuscular transmission involving acetylcholine led to the necessity to postulate an enzyme which hydrolysed it. This was done by Dale in 1914 . It was not until 1932, however, that Stedman, Stedman, and Easson were able to prepare from horse serum an enzyme which could actually do this and which was called cholinesterase. The enzyme was then found to be present in a wide variety of tissues and in muscle it was observed that more cholinesterase was present at the motor points than in other parts of the tissue (Marnay and Nachmansohn, 1937; Feng and Ting, 1938). Couteaux and Nachmansohn $(1938,1940)$ showed that after denervation a certain amount of cholinesterase remained in muscle homogenates, a finding which suggested that some part of the cholinesterase was located in the muscle itself and that the enzyme was not restricted entirely to the nervous elements in this tissue.

The precise region of localization of the enzyme had to await the development of a suitable histochemical technique. Such a technique was first described by Koelle and Friedenwald (1949). It was subsequently modified by Koelle (1951) to eliminate diffusion artefacts, and by Couteaux and Taxi (1952), Coërs (1953b), Gerebtzoff (1953), Gomori (1952), and others. Some of these latter authors introduced formalin fixation and the use of buffers at a lower $p \mathrm{H}$. With these technical aids it became possible not only to study the finer distribution of cholinesterase in the motor end-plate subneural apparatus but also to study the morphology of the apparatus. The motor end-plate was first described by Doyère in 1840 and was so named by Kühne (1886). Subsequent studies were made on it largely with the aid of silver and gold techniques by Tello (1917), Cuajunco (1942), Couteaux (1938, 1941), and Coërs (1955) among others. The author who contributed most to the study of the morphology of the subneural apparatus was Couteaux (1947) who, using stains such as dahlia violet and Janus green, demonstrated that this part of the end-plate was made up

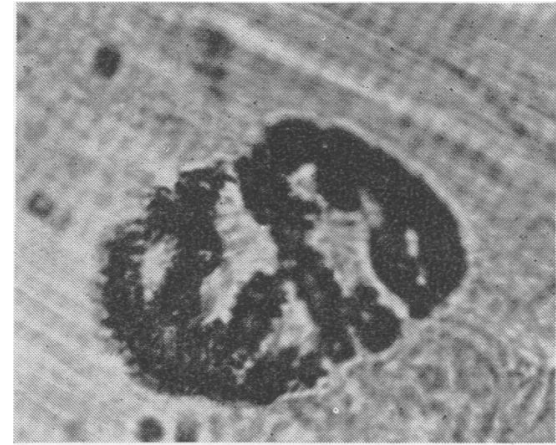

FIG. 1.- “ Classical " motor end-plates (as described by Koelle) in rat muscle. Technique used to demonstrate cholinesterase employed before formalin fixation, so that a clear morphological picture was obtained.

of a series of anastomosing gutter-like structures with transverse lamellae (Fig. 1), and suggested that this proved the existence of a delineating membrane (which he thought to be a continuation of the sarcolemma) between the neural and muscular portions of motor end-plates. This idea was confirmed by Couteaux and Coërs, among others, following the use of the cholinesterase techniques, and was further confirmed in electron microscope studies carried out by Robertson on lizard muscle (unpublished work quoted by del Castillo and Katz, 1956).

Very few observations have been made on the human subneural apparatus apart from those of Coërs $(1953,1955)$ on normal and pathological specimens of muscle.

The work to be reported in the present paper was carried out using Gomori's (1952) modification of Koelle and Friedenwald's (1949) original technique. The $p \mathrm{H}$ of the incubation medium for this technique is fairly high (about 6.5) and formalin fixation before incubation is not used. This means that minimal amounts of cholinesterase can be detected, but the 
morphological picture suffers somewhat from diffusion.

Our reason for choosing this method rather than that of Couteaux and Taxi (1952), Coërs (1953b), or Gerebtzoff (1953) was that we were attempting to demonstrate changes in cholinesterase activity in pathological, as compared with normal, human muscle. Various authors, including Taxi (1952) and Couteaux and Taxi (1952), have found, using biochemical and histochemical methods, that formalin inhibits cholinesterase activity to varying extents according to the site from which the cholinesterase is derived, and we ourselves have noticed that in human foetal muscle four hours' formalin fixation at $4^{\circ} \mathrm{C}$. causes total inhibition of cholinesterase activity at motor-end plates. It, therefore, seemed safer to eliminate formalin fixation despite its undoubted advantage in making the tissues easier to handle and in producing a clear morphological picture.

\section{Material}

Our series of specimens was taken from various sites in the body, but were predominantly specimens of gastrocnemius, deltoid, triceps or biceps, and comprised both normal and pathological muscle. The normal control muscles consisted of one specimen each of trapezius, deltoid, pectoralis major, quadriceps, and platysma taken from a young healthy adult within one hour after death; one specimen each of deltoid, gastrocnemius, triceps, and biceps taken within 12 hours of death from elderly people who died suddenly and had not suffered from any serious illness; one specimen of gastrocnemius taken during amputation of a leg which had not been in use for 25 years due to illness and accident; and one specimen of iliacus taken during cup arthroplasty of the hip for osteoarthritis of five years' standing.

The muscle taken from the amputated leg appeared histologically normal apart from some simple atrophy, but the specimen of iliacus contained scattered muscle fibres which showed basophilic degeneration with noticeable stringing and clumping of muscle nuclei. The muscle taken from the elderly people seemed to be healthy and normal apart from a tendency to vacuolation, and the fact that it was taken up to 12 hours after death is probably not of importance since Bergner and Durlacher (1951) found that cholinesterase remained stable in the human body for at least 26 hours after death, even if no special precautions had been taken to preserve it.

Our series of pathological muscle specimens numbered 33 and were derived from patients suffering from a variety of muscular and neuromuscular diseases; these included familial dystrophy, pseudo- hypertrophic muscular dystrophy, facio-scapulohumeral dystrophy, periodic paralysis, carcinomatous neuropathies and myopathies, polyneuritis and polymyositis, motor neurone disease, peroneal muscular atrophy, and several myopathies or neuropathies of unknown or doubtful aetiology. The biopsy was taken in each case from a muscle in which functional capacity was rapidly worsening at the time of biopsy, and in all cases the motor point was found both by stimulation on the skin surface and by direct stimulation of the exposed muscle.

\section{Technique}

Sections, each of $50 \mu$, of these muscles were incubated for a quarter and a half hour at $37^{\circ} \mathrm{C}$. in the incubation mixture as described by Gomori, containing $20 \mathrm{mg}$. acetyl thiocholine iodide $/ 10 \mathrm{ml}$. In most cases further sections were incubated for half an hour in a medium containing $24.8 \mathrm{mg}$. butyryl thiocholine iodide $/ 10 \mathrm{ml}$. instead of the acetyl compound. For a time this seemed to be an adequate method of control as it would detect any non-specific cholinesterase, but as there was the possibility that simple esterases might split the acetyl thiocholine, sections from about half of the specimens were incubated with the acetyl substrate and a concentration of $10^{-5} \mathrm{M}$ eserine salicylate or sulphate. As a further control, in a few cases, sections were also incubated with the butyryl substrate and eserine. Control sections from rat rectus femoris were always incubated with acetyl thiocholine and with acetyl thiocholine and eserine for half an hour.

In all cases, the sections were cut into Ringer or saturated sodium sulphate and were distributed randomly between the various incubation media. In this way the possibility of having sections with many end-plates incubated in some media and sections with few or no end-plates incubated in other media was eliminated. All sections were visualized according to the method described by Gomori, and after fixation in $10 \%$ neutral formol saline for half an hour to 24 hours, they were mounted in Apathy's medium. Mounting in Canada balsam proved impracticable for human muscle, although in rat muscle it improved the morphological picture considerably.

\section{Results}

In his papers published in 1953 and 1955, Coërs describes two basic types of end-plate structure to be found in normal human muscle. These are, first " terminaisons en plaque", that is, end-plates which have a ribbon-like subneural apparatus similar to that found in the rat, mouse, lizard, and other animals; and secondly " terminaisons en grappe" which seem to be characteristic of human muscle and are composed of small rounded areas of gutter aggregated together without any clear-cut connexion between them. The size of this second type of end-plate depends on the number of rounded areas of which they are composed. This number may vary from three or four to between 30 and 40 , 
so that at their largest these end-plates attain a considerable size (Figs. 2 and 3 ).

In addition, two other cholinesterase-positive structures have been described in human muscle and in the muscle of other animals. These are first the motor end-plates which are found at each end of the intrafusal fibres of muscle spindles, and secondly structures situated over the ends of muscle fibres at musculo-tendinous junctions. These latter were first described by Couteaux in 1953 in muscle of frog and mouse and fish, and have since been seen in a variety of other animals by Gerebtzoff and his co-workers (Gerebtzoff, 1956).

All of these types of cholinesterase-positive structures were present in both normal and pathological specimens of human muscle examined by the present authors. We found that the " terminaisons en plaque" or " classical " motor end-plates were arranged in rows across the muscle fibres (Fig. 4), as is seen in skeletal muscle of other animals, e.g., rat, mouse, and goat. These end-plates gave a stronger reaction for acetyl-cholinesterase than any other structure present, and the very rarely occurring cholinesterase-positive nerve fibres were always associated with them. There appeared to be no decrease of acetyl cholinesterase in these structures in any pathological condition.

The positive reaction obtained with acetyl thiocholine was completely inhibited by eserine in all muscles except for three patholagical specimens, each having a different clinical diagnosis, and in these three cases the residual eserine-insensitive enzyme was located in " classical " end-plates. These structures were also occasionally the site of non-specific cholinesterase. A strong reaction was found there by the present authors using butyryl thiocholine as substrate both in some of the control muscles and in seven of the pathological specimens (Fig. 5). The occurrence of a high concentration of this enzyme does not seem to be characteristic of any particular muscle or of any particular clinical picture, as far as we are able to judge from our limited series of specimens. In a further eight of our pathological muscles, a light non-specific cholinesterase reaction was obtained, which was sometimes associated with structures other than "classical" end-plates. The non-specific cholinesterase was always totally inhibited by eserine in the few cases where this control was carried out.

It is of interest perhaps to note here that perfect "classical" end-plates were found in a case of motor neurone disease (Fig. 6). Whether this was because the biopsy was of unaffected muscle fibres or whether in motor neurone disease the end-plates remain intact it is impossible to say at present. Only studies on a large series of muscle specimens taken from cases of motor neurone disease can settle this point.

Intact cholinesterase-positive musculo-tendinous junctions were found in several normal and abnormal muscle specimens (Fig. 7). These cholinesterasepositive structures consist of gutters arranged either parallel to each other or in a somewhat reticulate fashion to form a "cap" over the ends of muscle fibres. Similar structures were observed at points where muscle fibres appeared to end in the middle of a bundle. The reaction intensity for acetyl cholinesterase was less than in "classical" endplates, and seemed to be unaffected by pathological lesions, except in two cases, one of peroneal muscular atrophy and one of polyneuritis. On only one occasion did we find any non-specific cholinesterase at musculotendinous junctions. This was present in muscle from a case of pseudohypertrophic muscular dystrophy, and the reaction obtained was very slight. These observations therefore agree in the main with those of Coërs and Gerebtzoff, who found a weak true cholinesterase activity at these junctions.

In one or two specimens we saw the small motor end-plates on intrafusal fibres of muscle spindles, and, as far as we could tell from these few examples, these end-plates contained only true acetyl cholinesterase.

A variety of other structures containing acetyl cholinesterase were observed apart from the "terminaisons en grappe" of Coërs, which seem to constitute the most numerous form of end-plates in human muscle. Some of these other structures were discovered in pathological specimens of muscle, but as they showed no signs of disintegration, and other obviously normal end-plates were present, it is probable that these are also normal.

In several muscle samples structures consisting of parallel gutters arranged like a cake frill or pallisade were found. The gutters tended to be arranged somewhat closer together at the centre of the structure than at its periphery and occasionally the muscle fibre itself was slightly constricted at these points. This type of structure might very well represent some type of stretch receptor (Fig. 8).

Other cholinesterase-positive areas were also observed which consisted of parallel gutters arranged either parallel with or perpendicular to, the long axis of the muscle fibre, and in one case of facioscapulo-humeral dystrophy, structures were observed which were composed of a "shower" of parallel gutters with very well-marked transverse lamellae. Whether this latter arrangement also constituted a normal type of structure we would not like to say.

One other type of structure consisted of a spiral 


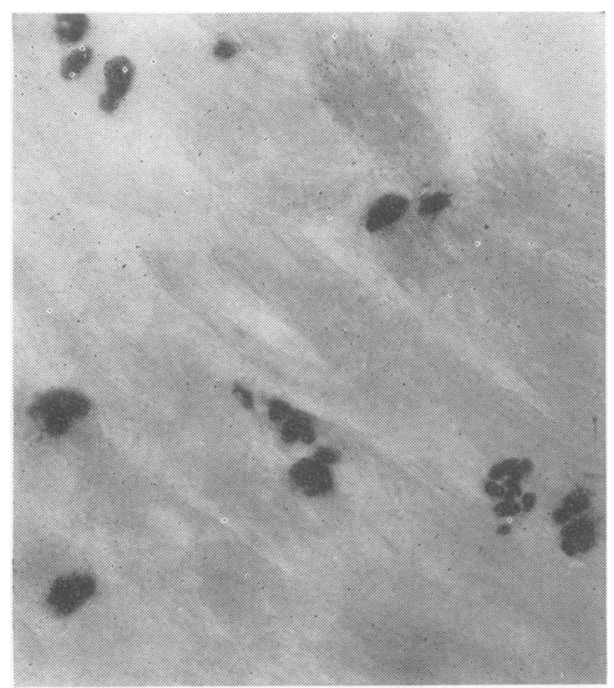

Fig. 2

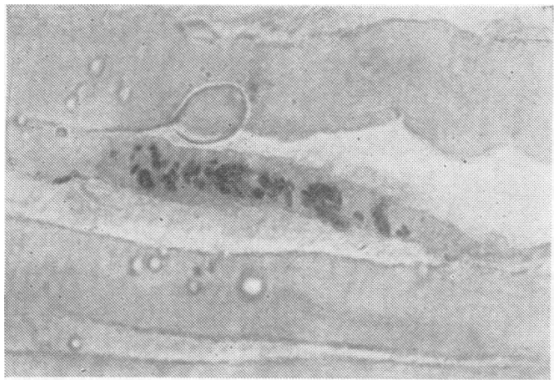

FIG. 3

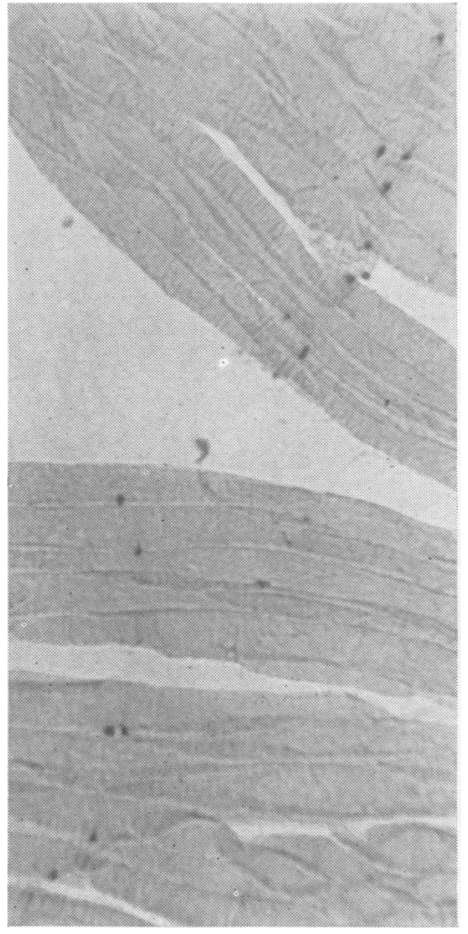

FIG. 4

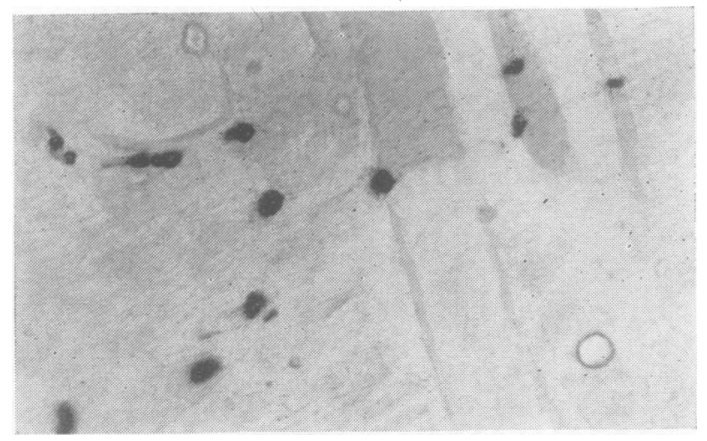

FIG. 5

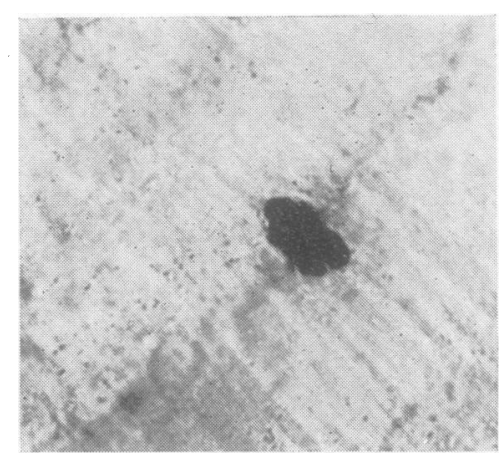

FIG. 6

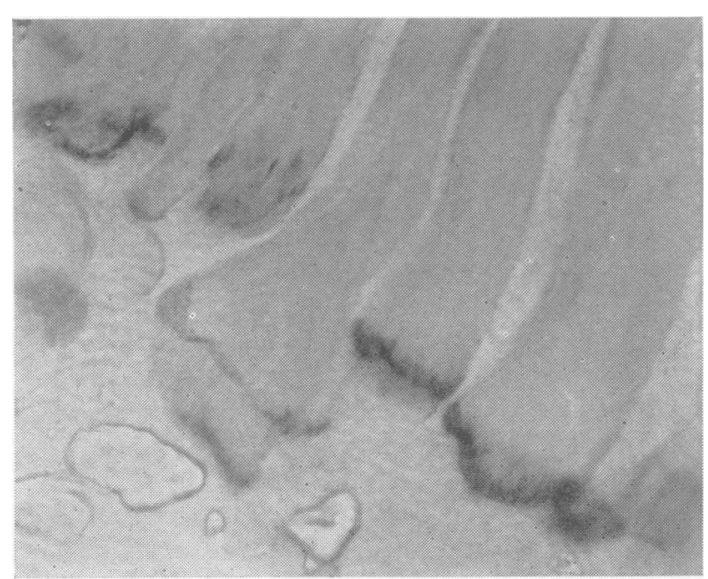

FIG. 7

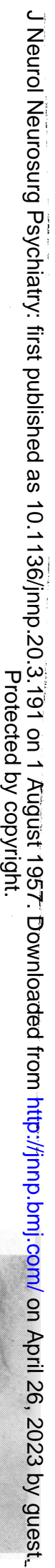

FIG. 8

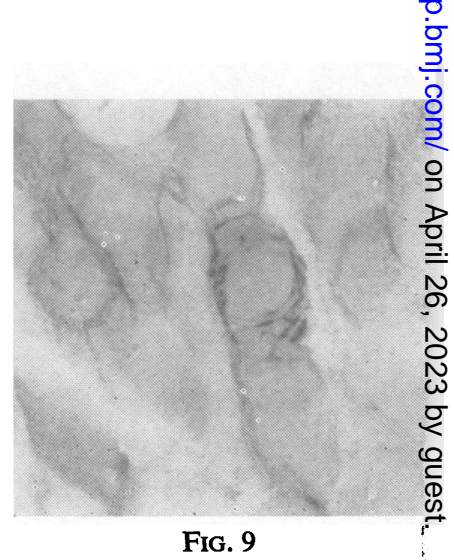


Fig. 2.-Semi-classical and small "dotted" end-plates in muscle taken from a case of chronic nephritis with non-specific neurological signs. These end-plates are probably normal.

Fig. 3.-Long " dotted " end-plate in muscle showing simple atrophy. This is probably a normal structure.

Fig. 4.-Line of "classical" end-plates in normal human muscle (No formalin fixation)

Fig. 5.-Group of " classical " end-plates obtained in human muscle using butyryl thiocholine (instead of acetylcholine) as substrate. The muscle was from a case of carcinomatous myopathy where there was no evidence of any histological abnormality in the muscle. In human muscle there appears to be an enzyme which can split butyryl thiocholine. It is present only in "classical" end-plates, and is not always found there. Its presence or absence is not correlated with the clinical diagnosis if muscle is obtained from a case of muscular disorder.

FIG. 6.-Single " classical" end-plate in muscle taken from a case of motor neurone disease.

Fig. 7.-Acetyl cholinesterase-positive structures at musculotendinous junctions in normal muscle.

FIG. 8.- “ Pallisade” cholinesterase-positive structure, probably a stretch receptor. This was present in muscle showing disuse atrophy but is probably a normal structure.

FIG. 9.-Motor end-plate in form of "spiral ". The muscle was taken from a case of ?? thyrotoxic myopathy, but the end-plate structure was probably normal.

gutter wound round a muscle fibre. The concentration of cholinesterase varied from place to place in the gutter of this structure (Fig. 9).

At what age these different types of end-plate develop is unknown. Coërs (1955) states that up to the age of 1 year in man the end-plates are of primitive form and that during the next 12 months they develop into " classical" or "dotted" endplates. End-plates of various forms have been found by the present authors in a boy of 9 years.

\section{Discussion}

A review of the results obtained in pathological specimens of muscle suggests that there is very little change in the cholinesterase-positive structures as compared with the normal. Certainly there was no decrease in the amount of cholinesterase present (except in the two instances cited before, where there was a decrease of enzyme in musculo-tendinous junctions) and in fact, if there was any change at all it was in the direction of an increase. Furthermore, except in one case of peroneal muscular atrophy where Coërs' (1955) findings were confirmed, it was not possible to say that there was any reduction in the number of cholinesterase-containing areas. In one example of pseudohypertrophic muscular dystrophy and one of facio-scapulo-humeral dystrophy, however, signs of end-piate structure breaking down were observed. In one of these cases (Fig. 10) this was particularly striking, since the

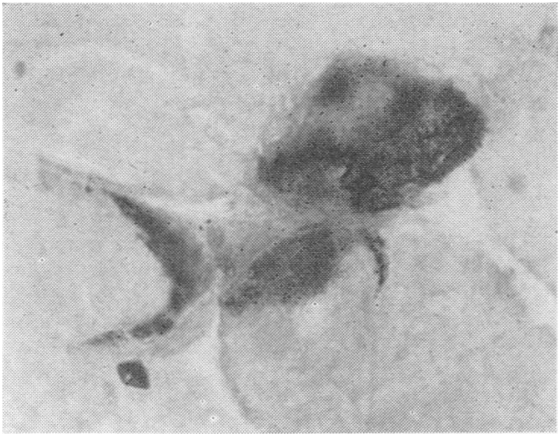

FIG. 10.-Motor end-plates undergoing degeneration. The muscle was from a case of facio-scapulohumeral dystrophy, but showed little morphological alteration.

clinical condition was of only a few months' st:nding and there was practically no change in muscle fibre structure. It is of interest that a control specimen also showed signs of what might have been motor end-plate disintegration. In a piece of iliacus, taken from a patient with osteoarthritis of five years' standing, there were, in some parts of the muscle fibres, numerous small dots of cholinesterasepositive material (Fig. 11). These "dots" were about the size of muscle nuclei, but were almost certainly not nuclei and were not artefacts either.

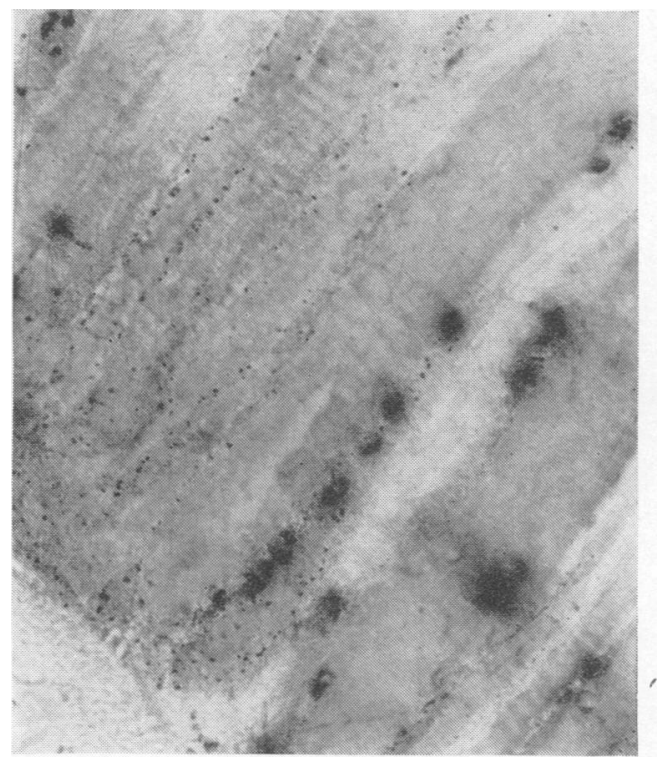

FIG. 11.-Cholinesterase in numerous structures of various types This was in muscle taken during cup arthroplasty of hip. The tiny dots are probably not nuclei. 


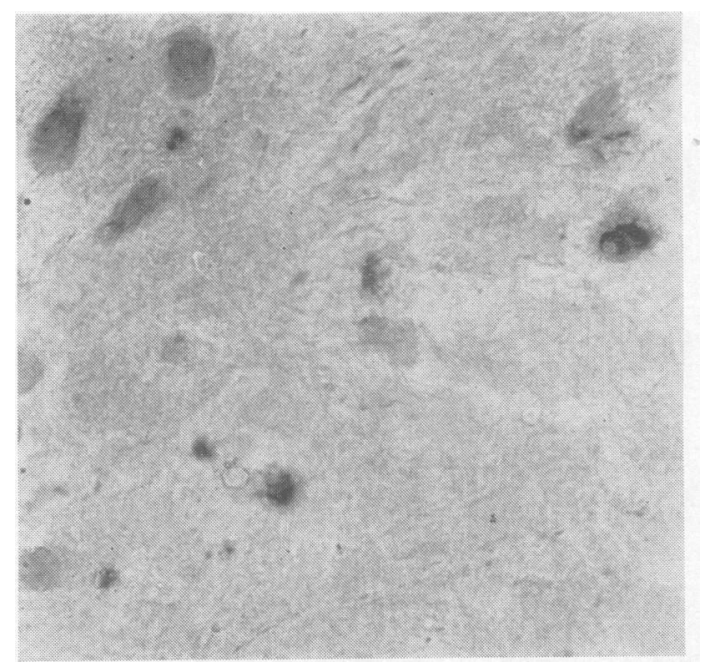

Fig. 12.-General view of muscle showing extreme atrophy of muscle fibres with replacement by dense connective tissue. This muscle was taken from a case of facio-scapulo-humeral dystrophy. Despite their extreme atrophy the muscle fibres still have on their surface numerous strongly cholinesterase-positive structures.

One of the most striking things which we have observed is that where there is extreme muscle fibre atrophy, almost to the point of total extinction of structure, the remaining pieces of fibre are almost completely covered with cholinesterase-positive gutters, sometimes very extensive and often seemingly normal in appearance (Fig. 12). This was particularly obvious in cases of pseudohypertrophic muscular dystrophy and familial dystrophy and facio-scapulo-humeral dystrophy, and it seems to us probable that the presence of cholinesterase and/or

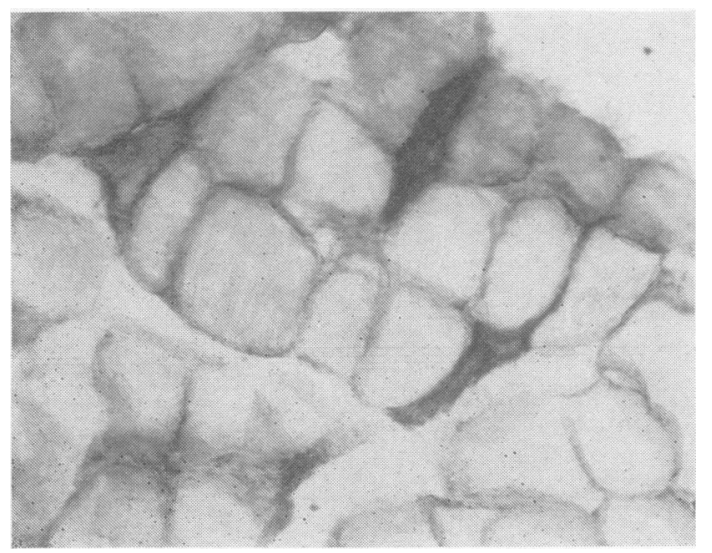

FIG. 13.-Muscle from a case of polymyositis, showing a stronger acetylcholinesterase reaction in atrophied degenerating muscle fibres than in the more normal fibres. its end-plate structure has some protective influence on the muscle fibre and prevents or delays its destruction.

Our preparations suggested that muscle fibres themselves contain large amounts of acetyl cholinesterase. The evidence for this is that, first a strong coloration is obtained when muscle sections are incubated in a medium containing acetyl thiocholine, and the depth of this colour bears no relationship to the number of end-plates in the section and is therefore not due to diffusion; furthermore, it still has the same intensity when no end-plates are present at all. This coloration of the muscle fibres is almost completely inhibited by eserine if this is added to the incubation medium; and the colour is absent when butyryl thiocholine is used as substrate. These observations are perhaps interesting in view of the fact that Couteaux and Taxi (1952) remark that it is odd that cholinesterase in the muscle substance is not disclosed by histochemical means. They suggest that this is due to lack of permeability of the sarcolemma, but it seems to us that it is more likely to be partly due to species difference and partly to technical differences.

Often where there was evidence of muscle atrophy with associated necrosis, there was an increase in the quantity of acetyl cholinesterase in the muscle substance (Fig. 13), which suggests that it may play some part in the processes of degeneration when it is present in increased amounts.

\section{Summary}

Several types of cholinesterase-positive structures are present in human muscle, not all of which are necessarily motor end-plates.

The only type of structure which contains nonspecific cholinesterase in any amount is the " classical " end-plate, or " terminaison en plaque" of Coërs. These end-plates also very occasionally contain some eserine-insensitive cholinesterase.

Signs of disintegration of end-plate structure are only rarely seen in muscular or neuromuscular disorders. There is very little evidence of decrease in cholinesterase activity in such diseases, and only occasionally does one find a decrease in number of cholinesterase-positive structures.

Cholinesterase-containing structures seem to exhibit a protective influence on muscle fibres, since often where there is extreme fibre atrophy, the parts which remain are covered with cholinesterasepositive structures.

There is a considerable amount of cholinesterase in the muscle substance, and an increase in this is often associated with atrophic necrosis of the muscle fibre. 
We are very much indebted to Dr. R. H. Henson and other members of the Neurology Department, The London Hospital, for their interest in this work and for arranging for biopsies to be taken from certain of their patients, and also to the theatre staff involved in the removal of these specimens.

\section{REFERENCES}

Bergner, A. D., and Durlacher, S. H. (1951). Amer. J. Path., 27, 1011. Castillo, J. del, and Katz, B. (1956). In Progress in Biophysics and Biophysical Chemistry, Vol. 6, p. 121. Pergamon Press, London.

Coërs, C. (1953a). Arch. Biol. (Paris), 64, 133.

(1953b). Rev. belge Path., 22, 306.

(1955). Acta neurol. psychiat. Belg., 55, 741

Couteaux, R. (1938). C.R. Soc. Biol. (Paris), 127, 218.

Couteaux, R. (1938). C.R. Soc.

(1947). Thèses presentées à la Faculté des Sciences, Université de Paris.
Couteaux, R. and Nachmansohn, D. (1938). Nature (Lond.) 142, 481.

- - (1940). Proc. Soc. exp. Biol. (N.Y.), 43, 177.

, and Taxi, J. (1952). Arch. Anat. micr., 41, 352

Cuajunco, F. (1942). Contr. Embryol. Carneg. Instn., 30, 127.

Dale, H. H. (1914). J. Pharmacol., 6, 147.

Doyère, L. (1840). Ann. Sci. nat. ser. 2 (Zool.), 14, 269.

Feng, T. P., and Ting, Y. C. (1938). Chin. J. Physiol., 13, 141.

Gerebtzoff, M. A. (1953). Acta anat. (Basel), 19, 366 .

Gomori. Extrait des Annales d'Histochimie, 1, 26. University of Chicago Press.

Koelle, G. B. (1951). J. Pharmacol., 103, 153.

- , and Friedenwald, J. S. (1949). Proc. Soc. exp. Biol. (N.Y.), $70,617$.

Kühne, W. (1886). Z. Biol., 23, 1.

Marnay, A., and Nachmansohn, D. (1937). C.R. Soc. Biol. (Paris), $125,41$.

Stedman, Edgar, Stedman, Ellen, and Easson, L. H. (1932). Biochem. J., 26,2056

Taxi, J. (1952). J. Physiol. (Paris), 44, 595.

Tello, J. F. (19i7). Trab. Lab. Invest. biol. Univ., Madr., 15,

The Effect of Insulin Coma and E.C.T. on the Three-Year Prognosis of Schizophrenia. D. M. Leiberman, J. Hoenig. and I. Auerbach.

\section{THE MAY (1957) ISSUE}

The May (1957) issue contains the following papers:-

The Entry of Radiosodium and of Bromide into Human Cerebrospinal Fluid. R. B. Bourdillon, M. Fiscker-Williams, Honor V. Smith, and K. B. Taylor.

Progressive Familial Choreoathetosis with Cutaneous Telangiectasia. Charles E. Wells and G. Milton Shy.

The Occurrence of Epileptic Fits in Leucotomized Patients Receiving Chlorpromazine Therapy. D. W. Liddell and N. Retterstöl.

The Urinary Excretion of Histamine in Schizophrenia. J. Adam and R. G. Mitchell.

Infantile Cerebral Gliosis with Giant Nerve Cells. L. Crome.

A Contribution to the Diagnosis of Tuberous Sclerosis. VL. Hudolin and F. Petrovčić.

Subdural Haematoma in an Adult after Air Encephalography. R. G. Robinson.

A Case of Brain Gumma. M. Bianchi and C. Frera.

Irregularities in the Contour of the Anterior Part of the Lateral Ventricle as Shown in Air Encephalograms. VL. Hudolin and F. Petrovčić.

Disorders of Oculomotor Function in Lesions of the Occipital Lobe. Morris B. Bender, Donald M. Postel, and Howard P. Krieger.

" The Limp Child ". John N. Walton.

Book Reviews.

A number of copies are still available and may be obtained from the Publishing Manager, British Medical Association, Tavistock Square, W.C.1, price $12 s .6 d$. 